\title{
Hi-GAL: The Herschel Infrared Galactic Plane Survey
}

S. Molinari, ${ }^{1}$ B. Swinyard, ${ }^{2}$ J. Bally, ${ }^{3}$ M. Barlow, ${ }^{4}$ J.-P. Bernard, ${ }^{5}$ P. Martin, ${ }^{6}$ T. Moore, ${ }^{7}$ A. Noriega-Crespo, ${ }^{8}$ R. Plume, ${ }^{9}$ L. Testi,${ }^{10,11}$ A. Zavagno, ${ }^{12}$ A. Abergel,${ }^{13}$ B. Ali,${ }^{14}$ P. André, ${ }^{15}$ J.-P. Baluteau, ${ }^{12}$ M. Benedettini, ${ }^{1}$ O. Berné, ${ }^{5}$ N. P. Billot, ${ }^{14}$ J. Blommaert, ${ }^{16}$ S. Bontemps, ${ }^{15,17}$ F. Boulanger, ${ }^{13}$ J. Brand,${ }^{18}$ C. Brunt, ${ }^{19}$ M. Burton, ${ }^{20}$ L. Campeggio, ${ }^{21}$ S. Carey, ${ }^{8}$ P. Caselli, ${ }^{22}$ R. Cesaroni, ${ }^{10}$ J. Cernicharo, ${ }^{23}$ S. Chakrabarti, ${ }^{24}$ A. Chrysostomou, ${ }^{25}$ C. Codella,${ }^{10}$ M. Cohen, ${ }^{26}$ M. Compiegne, ${ }^{27}$ C. J. Davis, ${ }^{25}$ P. De Bernardis, ${ }^{28}$ G. De Gasperis, ${ }^{29}$ J. Di Francesco, ${ }^{30}$ A. M. di Giorgio, ${ }^{1}$ D. Elia, ${ }^{131}$ F. Faustini, ${ }^{1}$ J. F. Fischera,${ }^{6}$ Y. Fukui,${ }^{32}$ G. A. Fuller,${ }^{33}$ K. Ganga, ${ }^{34}$ P. Garcia-Lario, ${ }^{35}$ M. Giard, ${ }^{5}$ G. Giardino, ${ }^{36}$ J: Glenn, ${ }^{3}$ P. Goldsmith, ${ }^{37}$ M. Griffin, ${ }^{38}$ M. Hoare, ${ }^{22}$ M. Huang, ${ }^{39}$ B. Jiang, ${ }^{40}$ C. Joblin, ${ }^{5}$ G. Joncas, ${ }^{41}$ M. Juvela, ${ }^{42}$ J. KirK, ${ }^{38}$ G. Lagache, ${ }^{13}$ J. Z. Li, ${ }^{39}$ T. L. LiM, ${ }^{2}$ S. D. LorD, ${ }^{14}$ P. W. LuCAS, ${ }^{43}$ B. Maiolo,${ }^{21}$ M. Marengo, ${ }^{24}$ D. Marshall, ${ }^{5}$ S. Masi, ${ }^{28}$ F. Massi, ${ }^{10}$ M. Matsuura, ${ }^{4}$ C. MenY, ${ }^{5}$ V. Minier, ${ }^{15}$ M.-A. Miville-Deschênes, ${ }^{13}$ L. Montier,${ }^{5}$ F. Motte, ${ }^{15}$ T. G. Müller, ${ }^{44}$ P. Natoli,${ }^{29}$ J. Neves, ${ }^{43}$ L. Olmi, ${ }^{10}$ R. Paladini, ${ }^{8}$ D. Paradis, ${ }^{8}$ M. Pestalozzi, ${ }^{2}$ S. Pezzuto, ${ }^{1}$ F. Piacentini, ${ }^{28}$ M. Pomarès, ${ }^{12}$ C. C. Popescu,,${ }^{45}$ W. T. Reach, ${ }^{8}$ J. Richer, ${ }^{46}$ I. Ristorcelli, ${ }^{5}$ A. Roy, ${ }^{6}$ P. Royer,${ }^{16}$ D. Russeil, ${ }^{12}$ P. Saraceno, ${ }^{1}$ M. Sauvage, ${ }^{15}$ P. Schilke, ${ }^{47}$ N. SchneiderBontemps, ${ }^{15}$ F. Schuller, ${ }^{47}$ B. Schultz, ${ }^{14}$ D. S. Shepherd, ${ }^{48}$ B. Sibthorpe, ${ }^{38}$ H. A. Smith, ${ }^{24}$ M. D. Smith, ${ }^{49}$ L. Spinoglio, ${ }^{1}$ D. Stamatellos, ${ }^{38}$ F. Strafella, ${ }^{21}$ G. Stringfellow, ${ }^{3}$ E. Sturm, ${ }^{44}$ R. Taylor, ${ }^{50}$ M. A. Thompson ${ }^{43}$ R. J. Tuffs,${ }^{51}$ G. Umana,${ }^{52}$ L. Valenziano, ${ }^{53}$ R. Vavrek, ${ }^{35}$ S. Viti, ${ }^{4}$ C. Waelkens, ${ }^{16}$ D. Ward-Thompson, ${ }^{38}$ G. White, ${ }^{2}$ F. Wyrowski, ${ }^{47}$ H. W. Yorke ${ }^{37}$ AND Q. ZHANG ${ }^{24}$

Received 2009 December 21; accepted 2010 January 14; published 2010 February 26

\begin{abstract}
Hi-GAL, the Herschel infrared Galactic Plane Survey, is an Open Time Key Project of the Herschel Space Observatory. It will make an unbiased photometric survey of the inner Galactic plane by mapping a $2^{\circ}$ wide strip in the longitude range $|l|<60^{\circ}$ in five wavebands between $70 \mu \mathrm{m}$ and $500 \mu \mathrm{m}$. The aim of Hi-GAL is to detect the earliest phases of the formation of molecular clouds and high-mass stars and to use the optimum combination of Herschel wavelength coverage, sensitivity, mapping strategy, and speed to deliver a homogeneous census of starforming regions and cold structures in the interstellar medium. The resulting representative samples will yield the variation of source temperature, luminosity, mass and age in a wide range of Galactic environments at all scales from massive YSOs in protoclusters to entire spiral arms, providing an evolutionary sequence for the formation of intermediate and high-mass stars. This information is essential to the formulation of a predictive global model of the role of environment and feedback in regulating the star-formation process. Such a model is vital to understanding star formation on galactic scales and in the early universe. Hi-GAL will also provide a science legacy for decades to come with incalculable potential for systematic and serendipitous science in a wide range of astronomical fields, enabling the optimum use of future major facilities such as JWST and ALMA.
\end{abstract}

Online material: color figures

\section{INTRODUCTION}

Dust is the most robust tracer of the "Galactic ecology"- the cycling of material from dying stars to the ionized, atomic, and

\footnotetext{
${ }^{1}$ INAF-Istituto Fisica Spazio Interplanetario, Rome, Italy; sergio.molinari@ ifsi-roma.inaf.it.

${ }^{2}$ STFC, Rutherford Appleton Laboratory, Didcot, UK.

${ }^{3}$ Center for Astrophysics and Space Astronomy (CASA), Department of Astrophysical and Planetary Sciences, University of Colorado, Boulder, CO.

${ }^{4}$ Department of Physics and Astronomy, University College London, UK.

${ }^{5}$ Centre d'Etude Spatiale du Rayonnement, CNRS, Toulouse, France.

${ }^{6}$ University of Toronto, CITA, Canada.
}

molecular phases of the ISM, into star-forming cloud cores, and back into stars. While atoms, ions, and molecules are imperfect tracers because they undergo complex phase changes, chemical

\footnotetext{
${ }^{7}$ Astrophysics Research Institute, John Moores University, Liverpool, UK. ${ }^{8}$ Spitzer Science Center, California Institute of Technology, Pasadena, CA. ${ }^{9}$ Department of Physics \& Astronomy, University of Calgary, Calgary, Canada.

${ }^{10}$ INAF-Osservatorio Astrofisico di Arcetri, Florence, Italy.

${ }^{11}$ European Southern Observatory, Garching bei Muenchen, Germany.

${ }^{12}$ Universitè de Provence, LAM, Marseille, France.

${ }^{13}$ Institut d'Astrophysique Spatiale, Universit Paris-Sud, Orsay, France.

${ }^{14}$ NASA Herschel Science Center, California Institute of Technology, Pasadena, CA.
} 
processing, and depletion onto grains, and are subject to complex excitation conditions, dust is relatively stable in most phases of the ISM. It is optically thin in the far-infrared (FIR) over most of the Galaxy, so that its emission and absorption simply depend on emissivity, column density, and temperature. Cold dust in particular $(10 \mathrm{~K} \leq \mathrm{T} \leq 40 \mathrm{~K})$ traces the bulk of nonstellar baryonic mass in all of these "habitats" of the Galactic ecosystem.

Temperature and luminosity and, as their byproduct, mass of cold dust measured over the entire Galactic plane (GP), are, at subparsec resolution, the critical observables needed to formulate a global predictive model of the cycling process between the Galactic ISM and star formation. This process drives the galactic ecology in normal spirals, as well as the enhanced star-formation rates of starburst galaxies and mergers, and a quantitative understanding of it is needed in order to follow the formation and evolution of galaxies throughout the cosmos. The adequate measurement of these key quantities has been beyond the capabilities of the previous mid- to far-infrared surveys of the Galactic plane (IRAS, Neugebauer et al. 1984; MSX, Price et al. 2001; COBE/DIRBE and FIRAS, e.g., Sodroski et al. 1994; ISO, Omont et al. 2003; Spitzer, Benjamin et al. 2003; Carey et al. 2009) either due to limited wavelength coverage and/or inadequate spatial resolution leading to confusion. The balloon-borne BLAST experiment (Pascale et al. 2008) implements Herschell SPIRE detector arrays and is providing exciting anticipations of what Herschel will do. The AKARI satellite (Murakami et al. 2007) improves over IRAS, and results from its FIR photometric mapping of the GP are eagerly awaited.

Observing the distribution and temperature of dust across the Galaxy will resolve many current debates such as the modes of formation of molecular clouds and high-mass stars.

Molecular clouds are traditionally thought to follow a "slow formation" scheme, where distributed material is accumulated by

\footnotetext{
${ }^{15}$ SAp CEA, Saclay, France.

${ }^{16}$ Institute for Astronomy, Katholieke Universiteit Leuven, Leuven, Belgium.

${ }^{17}$ Observatoire de Bordeaux, Bordeaux, France.

${ }^{18}$ INAF-Istituto di Radioastronomia, Bologna, Italy.

${ }^{19}$ School of Physics, University of Exeter, Exeter, UK.

${ }^{20}$ School of Physics, University of New South Wales, Sydney, Australia.

${ }^{21}$ Dipartimento di Fisica, Università del Salento, Lecce, Italy.

${ }^{22}$ School of Physics \& Astronomy, University of Leeds, Leeds, UK.

${ }^{23}$ Centro de Astrobiología, CSIC-INTA, Madrid, Spain.

${ }^{24}$ CfA, Harvard University, Cambridge, MA.

${ }^{25}$ Joint Astronomy Center, Hilo, HI.

${ }^{26}$ Radio Astronomy Lab, University of California, Berkeley, CA.

${ }^{27}$ Canadian Institute for Theoretical Astrophysics, University of Toronto, Toronto, Canada.

${ }^{28}$ Dipartimento di Fisica, Università di Roma 1 "La Sapienza," Rome, Italy.

${ }^{29}$ Dipartimento di Fisica, Università di Roma 2 "Tor Vergata," Rome, Italy.

${ }^{30}$ Herzberg Institute of Astrophysics, NRCC, Victoria, Canada.

${ }^{31}$ Observatòrio Astronomico de Lisboa, Lisboa, Portugal.

${ }^{32}$ Department of Astrophysics, Nagoya University, Nagoya, Japan.

${ }^{33}$ Jodrell Bank Centre for Astrophysics, School of Physics and Astronomy, University of Manchester, Manchester, M13 9PL, UK.

${ }^{34}$ APC, Université Paris 7, Paris, France.
}

large-scale perturbations such as the passage of a spiral arm. Shielding by dust and surface reactions on grains promotes the $\mathrm{HI} \rightarrow \mathrm{H}_{2}$ transition, which in turn allows the formation of other molecules that cool the cloud. Gravity, mediated by magnetic fields, leads to star formation. In this scenario cloud lifetimes are about $\sim 30 \mathrm{Myr}$ (Leisawitz et al. 1989). This picture has difficulty explaining the absence of quiescent, non-star-forming GMCs (however, see Palla \& Galli 1997) and the continuous regeneration of turbulence needed to support GMCs for many crossing times. Alternatively, a "fast formation" scenario has been proposed (Hartmann et al. 2001) in which most MCs are transient, short-lived structures (Stone et al. 1998; Padoan \& Nordlund 1999) created in the postshock regions of converging large-scale flows. Stars form on very short timescales (Elmegreen 2000). However, rapid MC formation requires rapid $\mathrm{HI} \rightarrow \mathrm{H}_{2}$ conversion (Goldsmith \& Li 2005). Accelerated $\mathrm{H}_{2}$ formation requires either high-density preshock conditions $\left(n \sim 200 \mathrm{~cm}^{-3}\right.$, $T \leq 100 \mathrm{~K}$; Price et al. 2001), or strong turbulence (Glover \& Mac Low 2007), higher than observed.

On the other hand, the formation of high-mass stars and of the star clusters hosting them is likely the most important process that shapes the formation and evolution of galaxies. Massive stars are responsible for the global ionization of the ISM. Their energetic stellar winds and supernova blast waves direct the dynamical evolution of the ISM, shaping its morphology, energetics and chemistry, and influencing the formation of subsequent generations of stars and planetary systems. Despite their importance, remarkably little is known about how massive stars form (McKee \& Tan 2003). We lack a "fundamental theory" or, rather, a galaxy-scale predictive model for star formation. One of the main limitations to this goal is the lack of statistically significant and well-characterized samples of young massive stars in the various evolutionary stages and environ-

\footnotetext{
${ }^{35}$ Herschel Science Center, ESAC/ESA, Madrid, Spain.

${ }^{36}$ RSSD, ESTEC/ESA, Noordwijk, The Netherlands.

${ }^{37}$ Jet Propulsion Laboratory, Pasadena, CA.

${ }^{38}$ School of Physics and Astronomy, Cardiff University, Cardiff, UK.

${ }^{39}$ National Astronomical Observatories, Chinese Academy of Sciences, Beijing, China.

${ }^{40}$ Department of Astronomy, Beijing Normal University, Beijing, China

${ }^{41}$ Département de physique, Université Laval, Québec, Canada.

${ }^{42}$ Helsinki University Observatory, University of Helsinki, Helsinki, Finland.

${ }^{43}$ Centre for Astrophysics Research, Science and Technology Research Institute, University of Hertfordshire, Hatfield, UK.

${ }^{44}$ MPE-MPG, Garching bei Muenchen, Germany.

${ }^{45}$ University of Central Lancashire, PR1 2HE, Preston, UK.

${ }^{46}$ Cavendish Labs, Cambridge, UK.

${ }^{47}$ MPIfR-MPG, Bonn, Germany.

${ }^{48}$ National Radio Astronomy Observatory, Socorro, NM.

${ }^{49}$ Centre for Astrophysics \& Planetary Science, University of Kent, Canterbury, UK.

${ }^{50}$ Center for Radio Astronomy, University of Calgary, Calgary, Canada.

${ }^{51}$ Max-Planck-Institut für Kernphysik, Heidelberg, Germany.

${ }^{52}$ INAF-Osservatorio Astrofisico di Catania, Catania, Italy.

${ }^{53}$ INAF-IASF, Bologna, Italy.
} 
ments on which a theory can be based. In turn, this results from the difficulty of gathering observational data on a large number of forming high-mass stars: they make up only a very small fraction of the total number of stars in the Galaxy, their early evolutionary phases of massive stars are more rapid than those of low-mass stars, they lie at large distance and form in crowded environments. There is thus a long list of questions that the community has been addressing for some time, not finding satisfactory answers. Here is an abridged list:

1. What is the temperature and density structure of the ISM? How do molecular clouds form and evolve, and how are they disrupted?

2. What is the origin of the stellar initial mass function (IMF)? What is its relationship to the mass function (MF) of ISM structures and cloud cores on all scales?

3. How do massive stars and clusters form and how do they evolve? What are the earliest stages of massive star formation and what are the timescales of these early phases?

4. How do the star-formation rate (SFR) and efficiency (SFE) vary as a function of galactocentric distance and environmental conditions such as the intensity of the interstellar radiation field (ISRF), ISM metallicity, proximity to spiral arms or the molecular ring, external triggers, and total pressure?

5. Does a threshold column density for star formation exist in our Galaxy? What determines the value of this possible threshold?

6 . What are the physical processes involved in triggered star formation on all scales and how does triggered star formation differ from spontaneous star formation?

7. How do the local properties of the ISM and the rates of spontaneous or triggered star formation relate to the global scaling laws observed in external galaxies?

Using the Herschel telescope, the largest ever in space, HiGAL will provide unique new data with which to address these questions. Hi-GAL will make thermal infrared maps of the Galactic plane at a spatial resolution 30 times better than IRAS and 100 times better than DIRBE, from which a complete census of compact source luminosities, masses, and spectral energy distributions (SEDs) will be derived. Source distances are a crucial parameter in this respect, and a dedicated effort will be needed (see $\S 4$ ). Extraction of statistically significant samples of starforming regions and cold ISM structures will be possible in all the environments of the Milky Way at all scales from massive young stellar objects (YSOs) in individual protoclusters to complete spiral arms.

In the following we present the specific characteristics of the survey as well as some of the science outcomes that we expect to obtain with this unique project.

\section{HI-GAL OBSERVING STRATEGY}

The area covered by Hi-GAL $\left(|l| \leq 60^{\circ},|b| \leq 1^{\circ}\right)$ contains most of the star formation in the Galaxy, and it is the one which
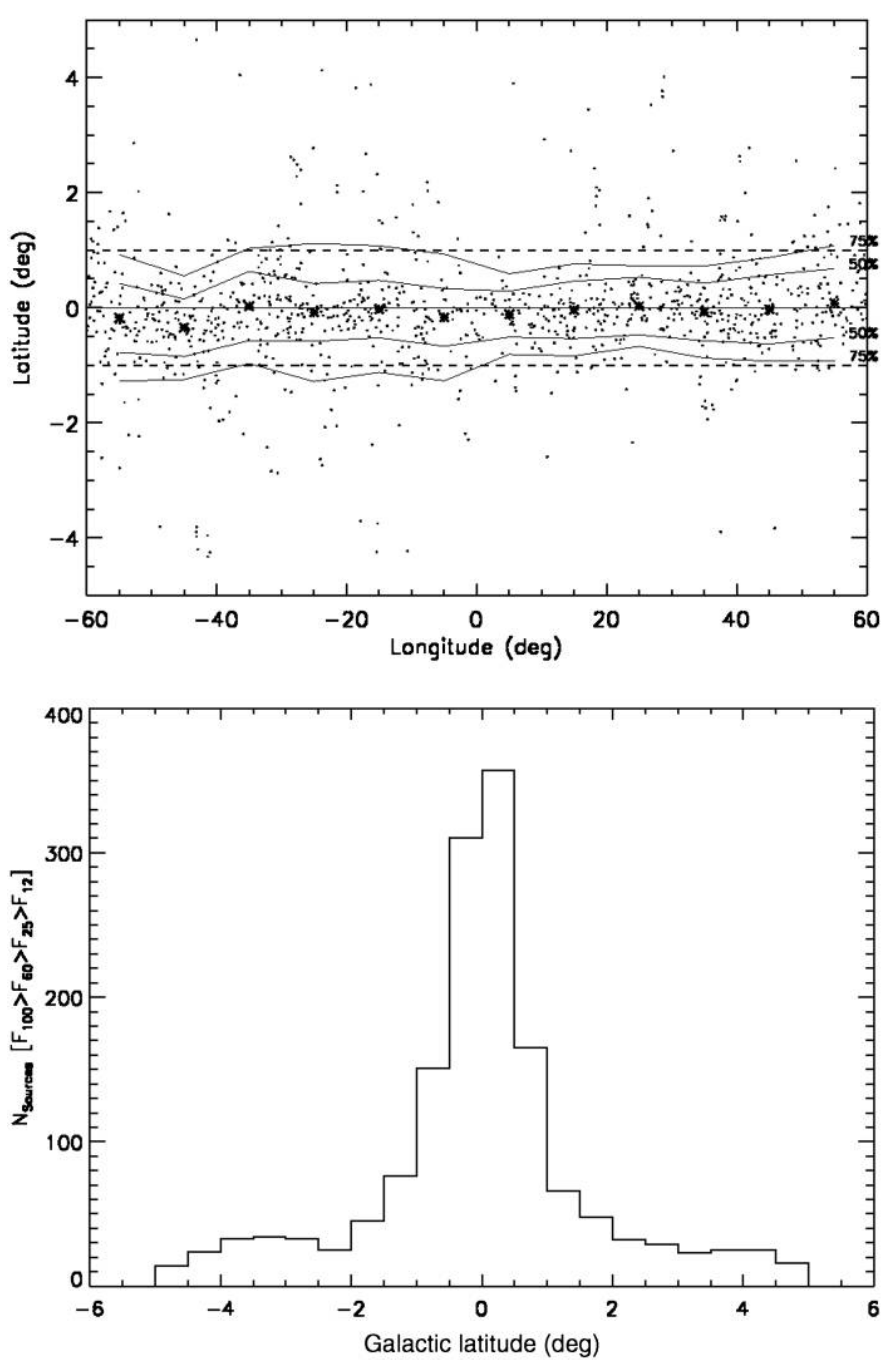

FIG. 1.-Top panel: $l-b$ plot of $\lambda$-rising SED IRAS sources; solid line is the Galactic midplane. The asterisks mark the median latitude of the sources computed in $10^{\circ} \mathrm{l}$ bins. The dashed lines delimit the regions where $50 \%$ and $75 \%$ of $|b| \leq 5^{\circ}$ IRAS sources are contained. Bottom panel: $b$-distribution of the same IRAS sources in the $|l| \leq 60^{\circ}$ region.

offers the best coverage in ancillary data which will be critical in the scientific analysis (see $\S 4$ ). The $b$ distribution and extent of the survey is shown in Figure 1 along with the $l-b$ plot of $\lambda$-rising SED IRAS sources $\left(F_{100}>F_{60}>F_{25}>F_{12}\right)$ which are potential YSOs. The Hi-GAL area (thick dashed lines in that figure) represents the $|b| \leq 1^{\circ}$ strip centered on the midplane and contains about $80 \%$ of all potential YSOs contained in $|b| \leq 5^{\circ}$ strip, thus encompassing most of the potential star-formation sites in the inner Galaxy.

The Herschel photometric cameras (PACS; Poglitsch et al. 2008) and SPIRE (Griffin et al. 2009) will be used in parallel mode $\left(\mathrm{pMode}^{54}\right)$ to maximize survey speed and wavelength

\footnotetext{
${ }^{54}$ In pMode, the Herschel telescope is scanning the sky in a raster fashion at constant speed, while both PACS and SPIRE acquire data simultaneously.
} 
coverage. Due to the instruments' wavelength-multiplexing capabilities, each pMode observation delivers maps at five different wavelengths: 70 and $170 \mu \mathrm{m}$ with PACS and 250, 350 and $500 \mu \mathrm{m}^{55}$ with SPIRE. Both cameras use bolometric detector arrays to map the sky by scanning the spacecraft along approximate great circles. Both instruments require their onboard subkelvin coolers to be recycled to provide the detectors with an operating temperature required of about $0.3 \mathrm{~K}$ in each case. In pMode, both instruments are placed into their photometric observing mode with the detectors at their correct operating

temperature, i.e., both instrument coolers are recycled, and data are taken from the five arrays simultaneously as the spacecraft is scanned across the sky.

The size and separation of the fields of view of PACS and SPIRE are shown in Figure 2 as viewed in the spacecraft coordinate system. Although the PACS array fully samples the point-spread function (PSF) spatially from the telescope, it still has gaps between the subarrays, and the SPIRE arrays only sparsely sample the sky. In order to make fully spatiallysampled maps it is necessary to scan the SPIRE array at an angle of $42.5^{\circ}$ with respect to its short symmetry axis. Scanning at an angle is also used for the PACS arrays to fill in for the gaps between subarrays. To achieve redundancy in the data and remove instrumental effects such as high-frequency detector response; slow drifts in gain; or stray light, saturation, and environmental (cirrus confusion) effects, it is also necessary to make at least a second pass over the same region of the sky using the other scan angle at $-42.5^{\circ}$, which, quite conveniently, is nearly orthogonal to the first one (see Fig. 3).

The distance between each scan in parallel mode is set by the size of the PACS array (being the smaller of the two), and the effective length of each leg of the raster takes into account the separation between the two fields of view. HSPOT, the Herschel-SPOT observing tool,$^{56}$ automatically calculates these parameters to ensure that the area required is covered. The distance between scans is approximately $155^{\prime \prime}$ and the excess length of the scan beyond the required length to cover the area is typically $20^{\prime}$. An example of how the sky is covered in a Parallel Mode observation used in Hi-GAL is shown in Fig. 3.

The strategy employed to cover the $-60^{\circ} \leq l \leq 60^{\circ},|b| \leq 1^{\circ}$ survey area is to conduct observations with series of $552.2^{\circ} \times$ $2.2^{\circ}$ square tiles with two passes over each tile at the two abovementioned scan angles (see Fig. 2). These tiles will be spaced every $2^{\circ}$, so that the overlap between tiles ensures that no coverage gaps are introduced by different tile orientation due to variable satellite roll angles with time; Figure 3 shows a section of

\footnotetext{
${ }_{55}$ The bandwidth of the filters are $60-85 \mu \mathrm{m}$ and $125-210 \mu \mathrm{m}$ for PACS (Poglitsch et al. 2008), while for SPIRE they are such that $\lambda / \Delta \lambda \sim 3$ (Griffin et al. 2009).

${ }^{56}$ At ftp://ftp.sciops.esa.int/pub/hspot/HSpot_download.html
}

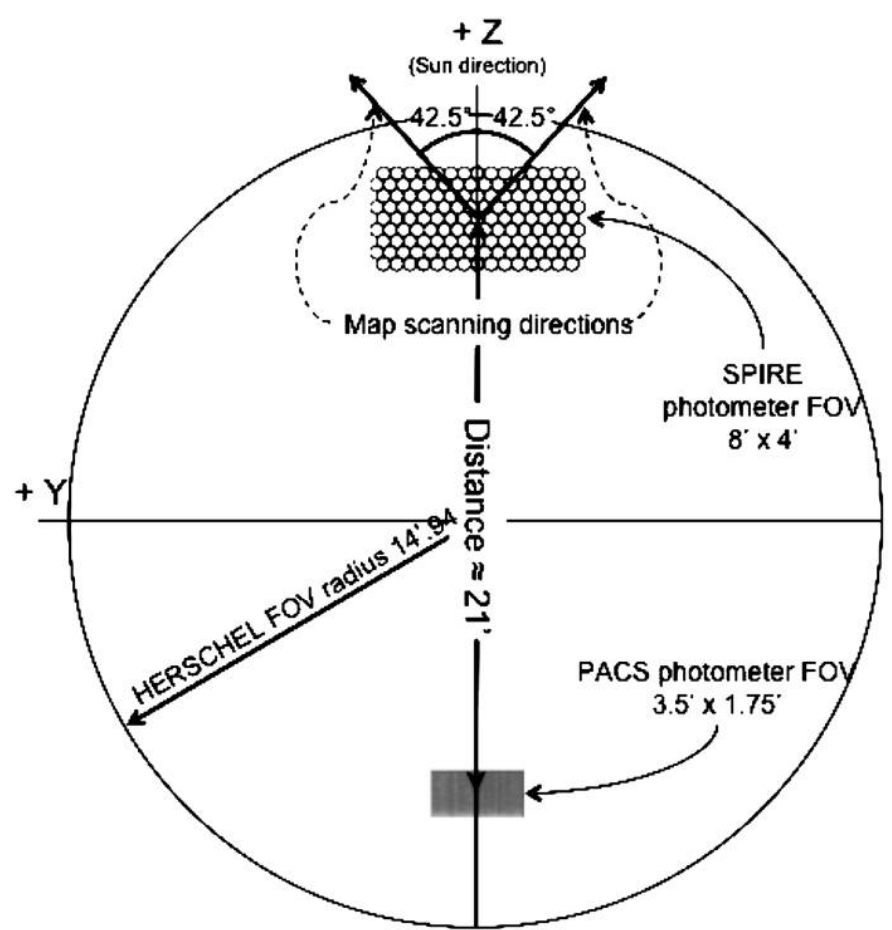

FIG. 2.-Field of view of the PACS and SPIRE instruments shown in the context of the Herschel field of view as viewed in the coordinate system of the spacecraft. $+Z$ refers to the axis toward the Sun. The $+\mathrm{X}$ axis is the telescope boresight and it would be oriented perpendicular to the plane of the page. The scan directions used to map the sky are as indicated. The different photometric channels of each instrument map the same region of the sky.

the galactic plane with consecutive observing blocks overlaid providing overlapping coverage.

Given the spatial separation required for PACS in the pMode observations, the SPIRE data is heavily oversampled and we

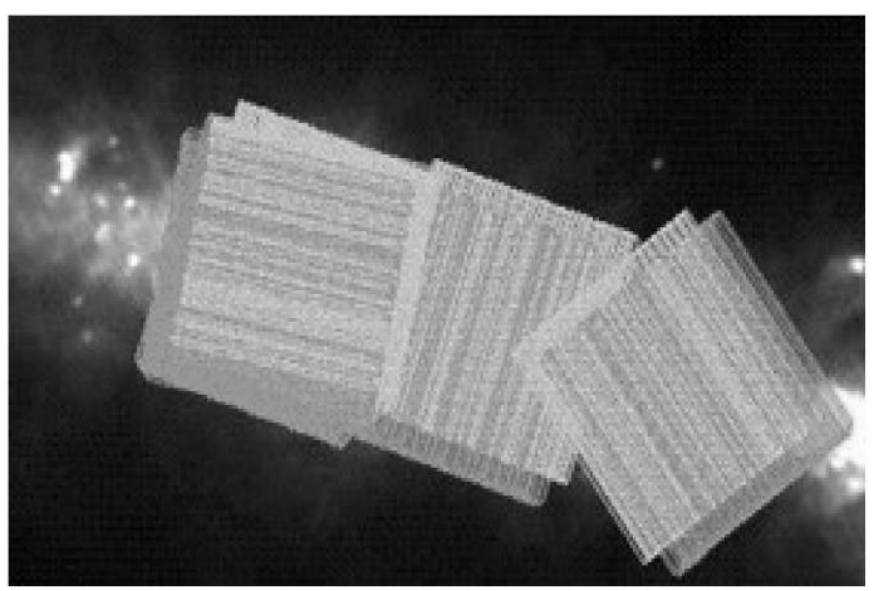

FIG. 3.-Sample AORs (Astronomical Observation Request) overlaid on the IRAS $100 \mu \mathrm{m}$ image of a portion of the Galactic plane. From left to right, the nominal and the orthogonal $2.2^{\circ} \times 2.2^{\circ}$ pMode AORs overlaid on one another. See the electronic edition of the PASP for a color version of this figure. 
cover a greater area than required with each individual instrument than would be required using them sequentially. Although it might seem that sequential PACS and SPIRE scan mode observations would be more efficient, in fact, the satellite overheads, set up, calibration and pointing acquisition, etc., require $30 \%$ more time to cover the same area sequentially compared to using the pMode.

In order to cover the maximum area in the shortest time, Hi-GAL data will be taken at the maximum possible scan speed for the satellite of $60^{\prime \prime} \mathrm{s}^{-1}$. This implies a beam-crossing time for the short wavelength, $250 \mu \mathrm{m}$ band of SPIRE of $3 \mathrm{~Hz}$ that is well within the bandwidth available in the detectors of $5 \mathrm{~Hz}$. However, although the PACS detectors have a similar response time, the much smaller PSF will be smeared out compared to that achievable with a slower scan. Additionally, because of the finite data transmission bandwidth between the Herschel satellite and the ground, it is necessary to perform onboard data compression for the PACS data which are the most demanding in terms of number of pixels (2048 for the $70 \mu \mathrm{m}$ array and 512 for the $170 \mu \mathrm{m}$ array) at the frame acquisition rate of $40 \mathrm{~Hz}$. The baseline configuration for the pMode is then to average on board groups of 8 frames at $70 \mu \mathrm{m}$ and 4 frames at $170 \mu \mathrm{m}$. Since the telescope is continuously scanning while acquiring, this co-addition will result in a further degradation of the PSF in the direction of the scan from its original diffractionlimited shape; the effect will be more severe at $70 \mu \mathrm{m}$ where the degradation should be of a factor of 2 based on simulations. This loss in imaging fidelity at the shortest wavelength is considered acceptable for a survey like Hi-GAL because, as discussed in $\S 1$, our main focus is toward a large-scale picture of the galaxy. Taking advantage of the orthogonal cross scan observing strategy, we may be able to recover some of the spatial resolution by careful deconvolution during postprocessing.

\subsection{Detection of Compact Sources}

The SPIRE digital readout electronics impose a limitation on the brightest sources that can be observed for a given offset setting (DC voltage removal) before digitization (SPIRE Instrument Users Manual, 2007). This problem can be alleviated to some extent by choosing a bias setting that gives the largest dynamic range per offset range. Simulations of the effect of bias variation show that setting a bias higher $(\sim 3 x)$ than the predicted nominal value will approximately double the dynamic range for most detectors under the conditions likely to be found in orbit (telescope temperature and emissivity and sky background). The same simulations show that a significant $(>10 \%)$ fraction of the SPIRE $250 \mu \mathrm{m}$ array detectors will saturate on sources greater than $500 \mathrm{Jy}$. The situation is slightly more relaxed for the 350 and $500 \mu \mathrm{m}$ arrays. We take the upper limit of detectable sources in the SPIRE bands as $500 \mathrm{Jy} \mathrm{beam}^{-1}$, assuming that a strong source instrument setting is used. This setting is required for all observations of bright regions/sources with SPIRE and is not a special Hi-GAL configuration. The saturation limits for PACS should be around $2000 \mathrm{Jy}$ at nominal bias, which will be used for the Hi-GAL survey.

The $1 \sigma$ sensitivities provided by HSPOT, for a single Astronomical Observation Request (AOR), are 17.6 and $26.8 \mathrm{mJy}$ in the two PACS 70 and $170 \mu \mathrm{m}$ bands, and 12.8, 17.6, and $14.9 \mathrm{mJy}$ for the SPIRE bands; co-addition of the orthogonal scanning patterns will provide $\sqrt{2}$ better figures. These sensitivities result from the adopted scanning strategy designed to maximize redundancy and map fidelity especially for largescale diffuse structures. However, the limiting factor for the detectability of sources and clouds will likely be cirrus confusion. Estimates based on recent BLAST measurements (Roy et al. 2010) suggest values on the order of 75,140 , and $160 \mathrm{mJy}$ in the 170,250 , and $500 \mu \mathrm{m}$ Herschel bands for a representative region of the Galactic plane at $l=45^{\circ}$; these values are greater than the detector sensitivities. Figure 4 shows the expected flux from a $20 M_{\odot}$ envelope as a function of distance (in $\mathrm{kpc}$ ) in each of the three above-mentioned bands, and for three different dust temperatures; we adopted $\beta=2$ and the dust opacity from Preibisch et al. (1993). The horizontal dashed lines (color coded with wavelength; see online article for color figures) represent the predicted confusion noises based on BLAST images. Figure 4 shows that we will detect the representative $20 M_{\odot}$ core everywhere in the Galaxy except for very cold dust $(T \leq 10 \mathrm{~K})$, for which detectability is predicted to be limited within a distance of about $5 \mathrm{kpc}$. We may then conclude that cirrus confusion is not going to be a problem for the investigations of the intermediate and high-mass star-formation studies that are the "core science" of this project (see $\S 3.3$ and 3.4).

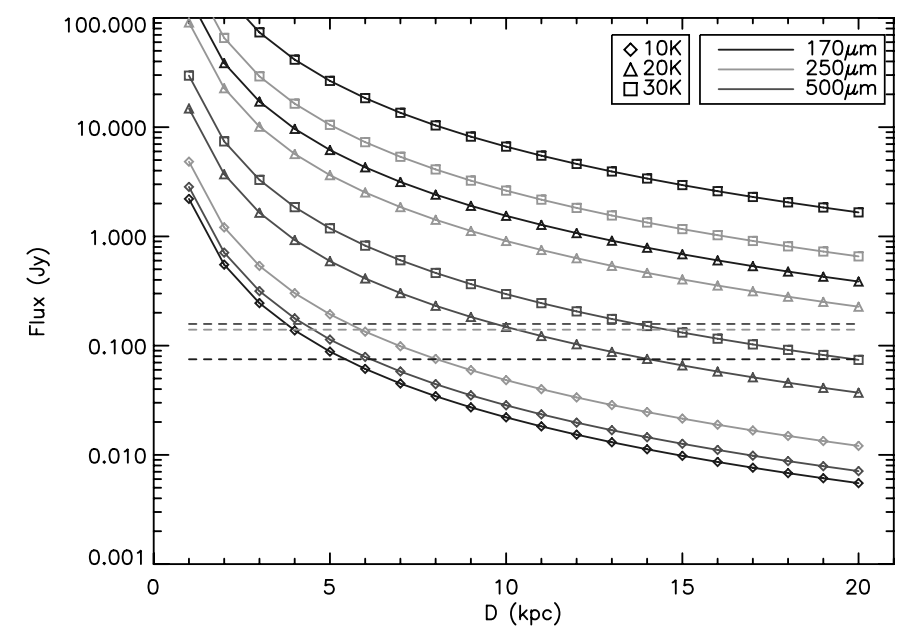

FIG. 4.-Flux expected in the 170, 250, and $500 \mu \mathrm{m}$ Herschel bands from a $20 M_{\odot}$ core (dust + gas), with key in upper right showing symbols representing different temperaturesand gray levels representing different wavelengths, as a function of distance in kpc. The dashed lines represent the confusion noise expected in the three bands. See the electronic edition of the PASP for a color version of this figure. 


\subsection{Detection of Extended Structures}

The diffraction-limited instrument beams at all wavelengths can be used to translate the confusion noises reported in the previous section into brightness units to investigate the detectability limits expected for diffuse extended structures. Calculations of the expected brightness levels from optically thin dust as a function of temperature and $\mathrm{H}$ column density (assuming gas $/$ dust $=100)$ are reported in Figure $5 \mathrm{a}$ and $5 \mathrm{~b}$ for $\lambda=170$ and $500 \mu \mathrm{m}$, respectively, where the expected confusion noise levels are also reported in red lines.

The figures show that with a typical confusion noise of about $10 \mathrm{MJy} \mathrm{sr}^{-1}$ at $500 \mu \mathrm{m}$, it will be possible to detect most Infrared Dark Clouds (IRDCs, see § 3.2), where column densities are in the range $10^{23}-10^{25} \mathrm{~cm}^{-2}$. Less dense clouds with column densities of the order of few $10^{21} \mathrm{~cm}^{-2}$ should be easily detectable at levels of tens of MJy sr ${ }^{-1}$ at $170 \mu \mathrm{m}$ at temperatures as low as $T \sim 20 \mathrm{~K}$. The situation may be less simple in the regions closer to the Galactic center. However, our broad spectral coverage provides an important advantage for measuring the temperature accurately, and for isolating structures and sources with temperature different from the standard diffuse ISM cirrus $(\sim 20 \mathrm{~K})$. Besides, all of these quantities are based on extrapolated estimates and we will provide the definitive measurement of the cirrus confusion at Herschel wavelengths and resolution.

\subsection{Checks on Photometric Calibration}

Virtually every block of the Hi-GAL survey will contain secondary calibrators, either stars or other well-known objects, ensuring accurate checks of the flux calibration of Hi-GAL data. Our baseline calibration targets will be the 400 stars used as calibrators for the Spitzer GLIMPSE-I/II surveys. These are mostly A0-5V or K0-M0III stars, although they also include $\sim 60$ calibrators of hot or warm dwarfs (B-G). In order to remove sources with nonphotospheric FIR emission, we will make predictions for the $24 \mu \mathrm{m}$ fluxes and then test for excesses in the MIPSGAL data first. Once anomalous sources are excluded we can extrapolate to the FIR and create an initial set of calibrators.

We will also be able to obtain a reliable calibration for extended sources, which is one of the more difficult parts of the nominal instrumental calibration activity. As part of the calibration scheme we will compare fluxes in the SPIRE $500 \mu \mathrm{m}$ band with fluxes from the same band of Planck-HFI in suitable locations. ${ }^{57}$

\footnotetext{
${ }^{57}$ Many of the Hi-GAL Co-Is are also Planck Consortium members.
}

\section{HI-GAL KEY SCIENCE}

\subsection{The Distribution of the ISM Temperature and the Intensity of the Interstellar Radiation Field}

At near-infrared wavelengths, the emission from dust is produced by small particles (size 1.2-15 $\mu \mathrm{m}$ ), whose abundance varies significantly, being strongly depleted by coagulation processes in the dense ISM (e.g., Bernard et al. 1993; Abergel et al. 1994; Stepnik et al. 2003). Far-infrared (FIR) emission is produced by larger grains (size $15-110 \mu \mathrm{m}$ ), which are more stable and dominate the total dust mass, and trace all phases of the ISM. The ISM dust spectrum peaks in the FIR where the Galaxy is transparent. FIR emission is therefore a reliable tracer of the overall ISM column density structure in our Galaxy. Other phaseindependent tracers include dust absorption and gamma-ray production; however, the former can be used to sample only the nearest $1 \mathrm{kpc}$, and gamma-ray surveys currently lack sensitivity and angular resolution.

Variations in the FIR emissivity (the ratio of surface brightness to column density) are dominated by the nonlinear effects of dust temperature through the Planck function. Fortunately, the shape of the dust SED as measured by PACS and SPIRE will be most sensitive to temperature variations as the spectral bands sample the peak of the Big Grain emission, and the contribution of Very Small Grains can be estimated from the Hi-GAL data at $70 \mu \mathrm{m}$ and MIPSGAL at $24 \mu \mathrm{m}$. The dust temperature $\left(T_{\mathrm{d}}\right)$ and its spatial variations will therefore be measured precisely. This important parameter can be used, in conjunction with complementary data from Planck, HI, CO, $\mathrm{H} \alpha$, and gamma-ray surveys, to estimate the strength and spectral shape of the InterStellar Radiation Field (ISRF), which is set by the stellar content in a given region. So far, the dust temperature in the Galactic plane has been mapped over limited regions using IRAS (Kim et al. 1999, Douglas \& Taylor 2007, and over the entire plane at a resolution of 40' (Lagache et al. 1998) using DIRBE. Hi-GAL will improve with respect to the latter by a factor of about 100 in linear scales. It will trace the local radiation field on scales relevant to star formation, and provide mass estimates even at large distances. In the case of the dense medium, determining the 3D distribution of the ISRF strength and spectral shape in a given cloud will require radiative transfer modeling. This is possible, even for complex geometries, using Monte Carlo codes (e.g., Juvela \& Padoan 2003). Using such codes, the equilibrium dust temperature $T_{\mathrm{d}}$ and the dust emission can be predicted at any $3 \mathrm{D}$ location in the cloud. Integration along the line of sight in turn allows us to predict $2 \mathrm{D}$ emission maps.

\subsection{Molecular Cloud Formation}

About a quarter of the mass in the ISM is in molecular form (Blitz 1997) and most of that material resides in giant molecular clouds (GMCs). Since GMCs are also the dominant sites of star 

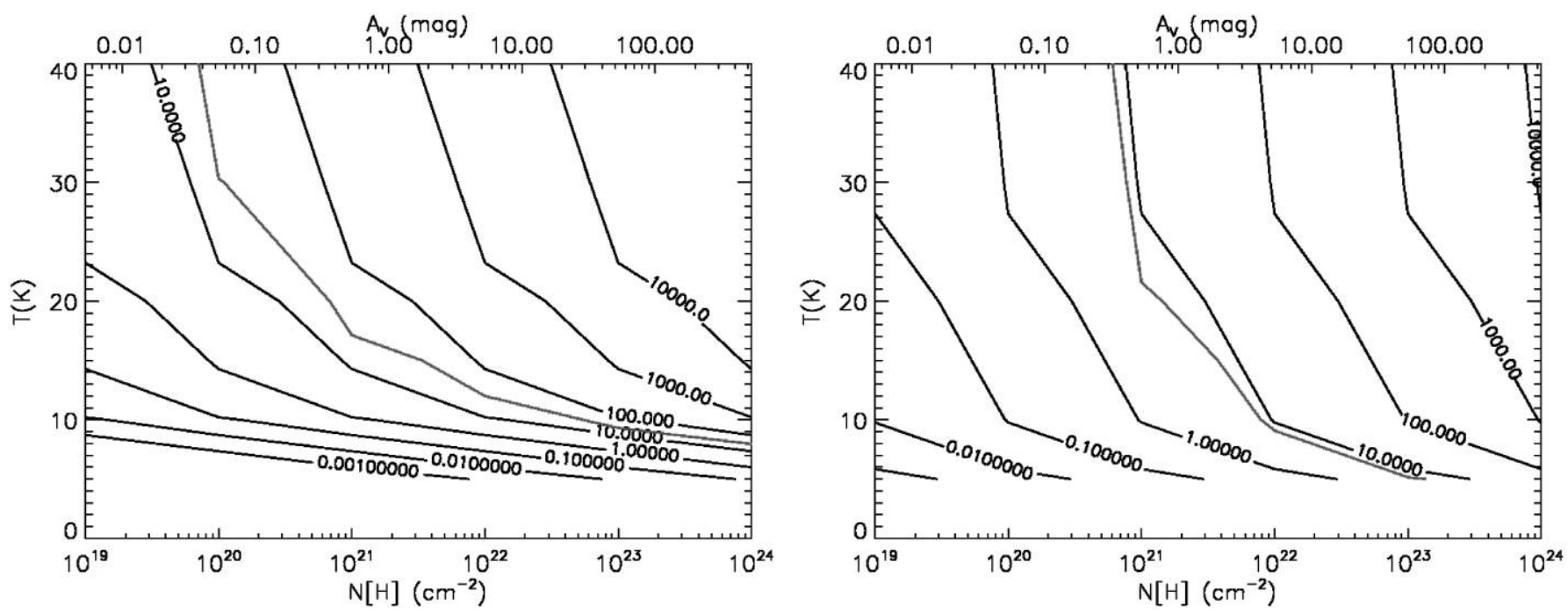

FIG. 5.-Brightness (in MJy sr ${ }^{-1}$ ) of optically thin dust as a function of temperature and column density at $170 \mu \mathrm{m}($ left) and $500 \mu \mathrm{m}($ right). The gray lines indicate the expected confusion noise. See the electronic edition of the PASP for a color version of this figure.

formation, understanding their origins and evolution is essential to our understanding of the Galactic environment.

In combination with molecular line surveys, Hi-GAL will provide the data needed to derive basic physical properties of GMCs. We will detect and characterize cold structures in the inner GP and classify them based on star-formation activity. Detection statistics for clouds with different temperatures and degree of star-formation activity will provide the fraction of quiescent versus star-forming clouds. It will thus be possible to constrain the properties and lifetimes of GMCs in our Galaxy and to compare with the predictions of fast evolution of molecular clouds (Hartmann et al. 2001) or a more traditional slow evolution of star formation in our Galaxy (Shu et al. 1987). Variations with galactocentric radius will determine if the slow/fast scenarios are mutually exclusive or reflect different initial/ environmental conditions. A large-area survey like Hi-GAL will provide the needed statistical significance in all mass bins, especially at the high-mass end, and in a variety of Galactic environments.

Direct detection of cold (i.e., $T<20 \mathrm{~K}$ ) dust, which could be the quiescent counterparts to GMCs, has been difficult in the past (Sodroski et al. 1994; Reach et al. 1995; Lagache et al. 1998; Kim et al. 1999; Douglas \& Taylor 2007) either because of insufficient wavelength coverage (e.g., IRAS) or inadequate spatial resolution (DIRBE, FIRAS). CO observations are problematic due to molecular freeze-out onto grains (Flower et al. 2005), or photochemical effects in low-metallicity environments (Bot et al. 2007). The recent detection of very cold clumps in the GP with Archeops (Désert et al. 2008) and BLAST (Olmi et al. 2009), confirms the FIR and submillimeter continuum as the best tool to trace cold ISM components. Notable examples are Infrared Dark Clouds (IRDCs) and HI Self-Absorption (HISA) clouds.

IRDCs are structures initially discovered as extinction features against the bright mid-IR Galactic background, and soon verified to exhibit properties similar to molecular clouds. Their properties $\left(n>10^{5} \mathrm{~cm}^{-3}, \mathrm{~N}_{\mathrm{H}} \sim 10^{22}-10^{24} \mathrm{~cm}^{-2}\right.$, and $T<$ $25 \mathrm{~K}-$ Egan et al. 1998; Carey et al. 2000, $R \sim 5 \mathrm{pc}$, and $M \sim 10^{3} M_{\odot}$-Simon et al. 2006; Rathborne et al. 2006) suggest that they are the precursors of cluster-forming molecular clumps like Orion. Thus, IRDCs are ideal for the study of the pristine, undisturbed physical conditions that may produce massive stars and clusters. IRDCs have only been detected against the bright Galactic mid-IR background (mostly for $|l| \leq 30^{\circ}$ ); their true Galactic distribution is unknown. Modeling of IRDCs in the IR and submillimeter (Fig. 6, Stamatellos et al. 2004) proves Herschel's unique ability to detect them and to measure their SEDs. IRDCs, with $\tau_{200 \mu \mathrm{m}} \geq 1$, are not detectable by either IRAS or Spitzer. Hi-GAL will provide a definitive inventory of cold dust and potential sites of massive star cluster formation everywhere in the inner GP.

HISAs are traced by cold $\mathrm{H}$ I gas seen in absorption against a background of warm H I emission (Gibson et al. 2000, 2005; Goldsmith \& Li 2005); they may provide additional clues to the formation of molecular clouds. When compared with molecular tracers, these cold (relative to the ambient neutral medium) and relatively quiescent ( $\Delta v \sim 1-3 \mathrm{~km} \mathrm{~s})$ clouds show a wide range of $\mathrm{HI} / \mathrm{H}_{2}$ ratios (Li \& Goldsmith 2003; Klaassen et al. 2005) which suggests that they might be $\mathrm{HI} \rightarrow \mathrm{H}_{2}$ conversion sites. Detailed studies of their FIR $\rightarrow$ mm SED shapes can help clarify this issue, providing evidence for the grain types necessary for a reasonable $\mathrm{HI} / \mathrm{H}_{2}$ formation timescale (Goldsmith \& Li 2005). 

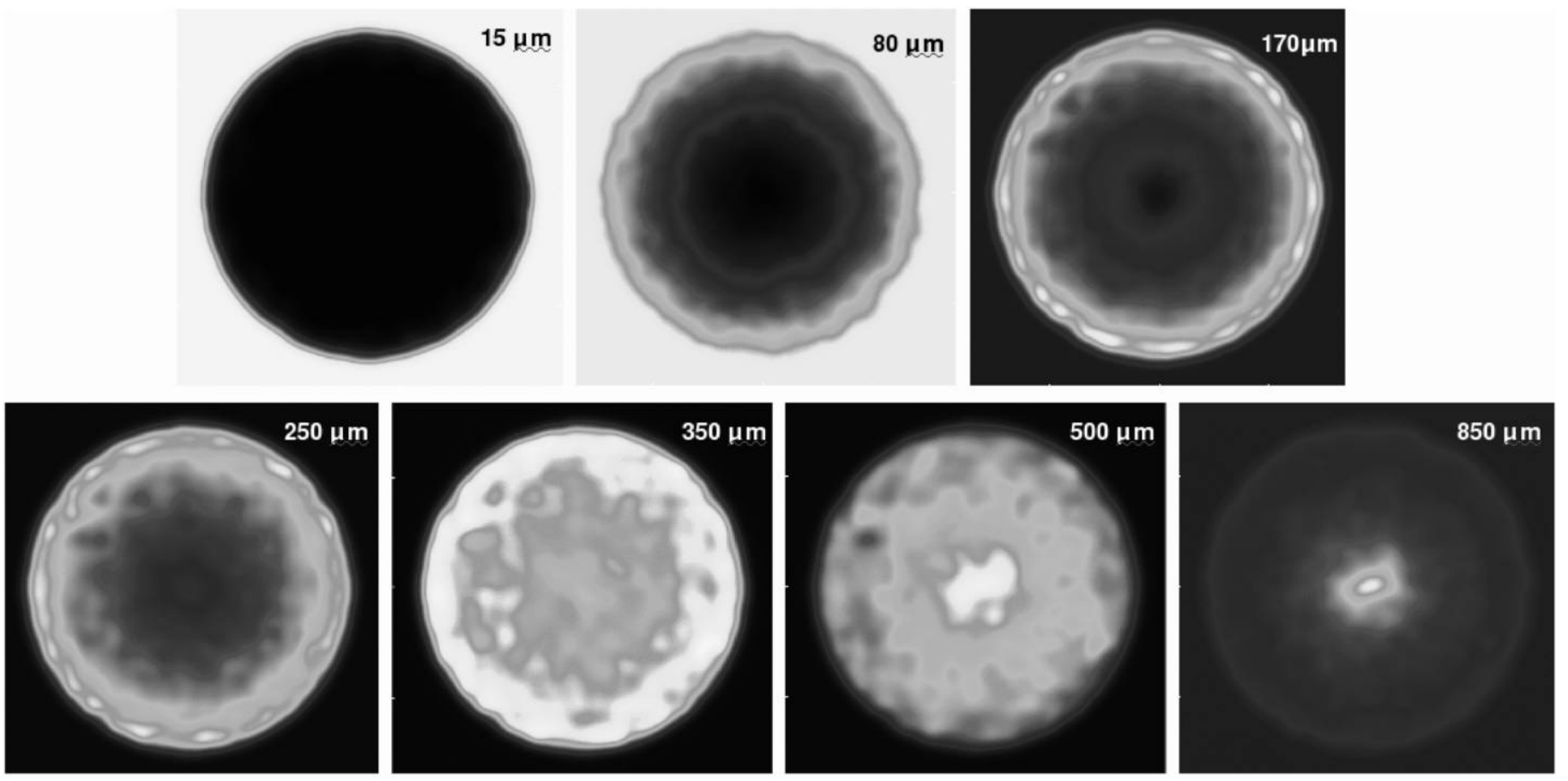

FIG. 6.-Model of a typical IRDC after Stamatellos et al. (2004) at seven different wavelengths illustrates the importance of the Herschel wavelength coverage and resolution (at $2 \mathrm{kpc}$ the linear extent is $5^{\prime}$ ) to obtain measurements of IRDCs in emission. See the electronic edition of the PASP for a color version of this figure.

\subsection{Timeline of High-Mass Star Formation}

The paradigm for the formation of solar-type stars via accretion through a circumstellar disk (Shu et al. 1987) predicts an evolution from cores to protostars and, finally, pre-main-sequence stars that is well matched with distinctive characteristics of their SEDs (Lada \& Wilking 1984; Andre et al. 1993). The empirical classification of the SED of low-mass YSOs has thus been used as a powerful tool to constrain theoretical models.

Higher mass stars reach the conditions for H-burning faster than the time required to assemble them, so that winds and radiative feedback will strongly influence accretion and may limit the final mass of the star (Zinnecker \& Yorke 2007). However, since massive stars exist, several theories have been proposed to solve this puzzle, including accretion from a disk, a very highpressure ambient medium, the "flashlight effect" (Yorke \& Sonnhalter 2002; McKee \& Tan 2003), competitive accretion, and coalescence (e.g., Stahler et al. 2000). Application of SEDbased classification tools, and evolutionary diagnostics like the $M_{\text {env }}-L_{\text {bol }}$ diagram which relates the bolometric luminosity of a YSO to the mass of its envelope (Molinari et al. 2008), to a large sample of luminous protostar candidates in the GP will define a timeline for the various phases of massive star formation that will constrain the theories and lead to new estimates of the SFR. Clearly the source distance is the crucial parameter here; we are collecting the information from the major molecular line surveys over the inner Galactic plane, while planning to undertake additional surveying activities at a variety of facilities in several high-density tracers to get additional data (see $\S 4$ for more detail).

An evolutionary sequence for massive YSOs has been proposed in which cold massive cloud cores evolve into Hot Molecular Cores with outflow, IR-bright massive YSO, and finally into ultracompact (UC) H II regions (e.g., Evans et al. 2002; Kurtz et al. 2000), but it is qualitative and based on small and possibly incomplete samples. Samples of bright and massive YSOs (Molinari et al. 1996; Sridharan et al. 2002; Hoare et al. 2004) are IRAS or MSX selected and tend to suffer from age biases and confusion which prevent firm quantitative conclusions. A phase of intense and accelerating accretion prior to H-burning ignition, that may be observable (e.g., Molinari et al. 1998) in the form of dense condensations devoid of IR as well as radio continuum emission, seems confirmed by recent large millimeter surveys (Beltrán et al. 2006; Hill et al. 2005). Millimeter continuum alone, however, cannot distinguish between precollapse condensations and rapidly accreting cores; HiGAL will use the full potential of Herschel wavelength coverage and spatial resolution to trace the SED peak of dust envelopes in all phases, from massive prestellar condensations to ultracompact (UC) H II regions. An angular resolution of 30" or less, typical of Hi-GAL, has been proven (e.g., Molinari et al. 2008) to be the key to building accurate SEDs, deriving reliable luminosities for massive YSOs, and distinguishing embedded UC H IIs strongly emitting in the mid-IR and radio from preUC H IIs (Fig. 7). 

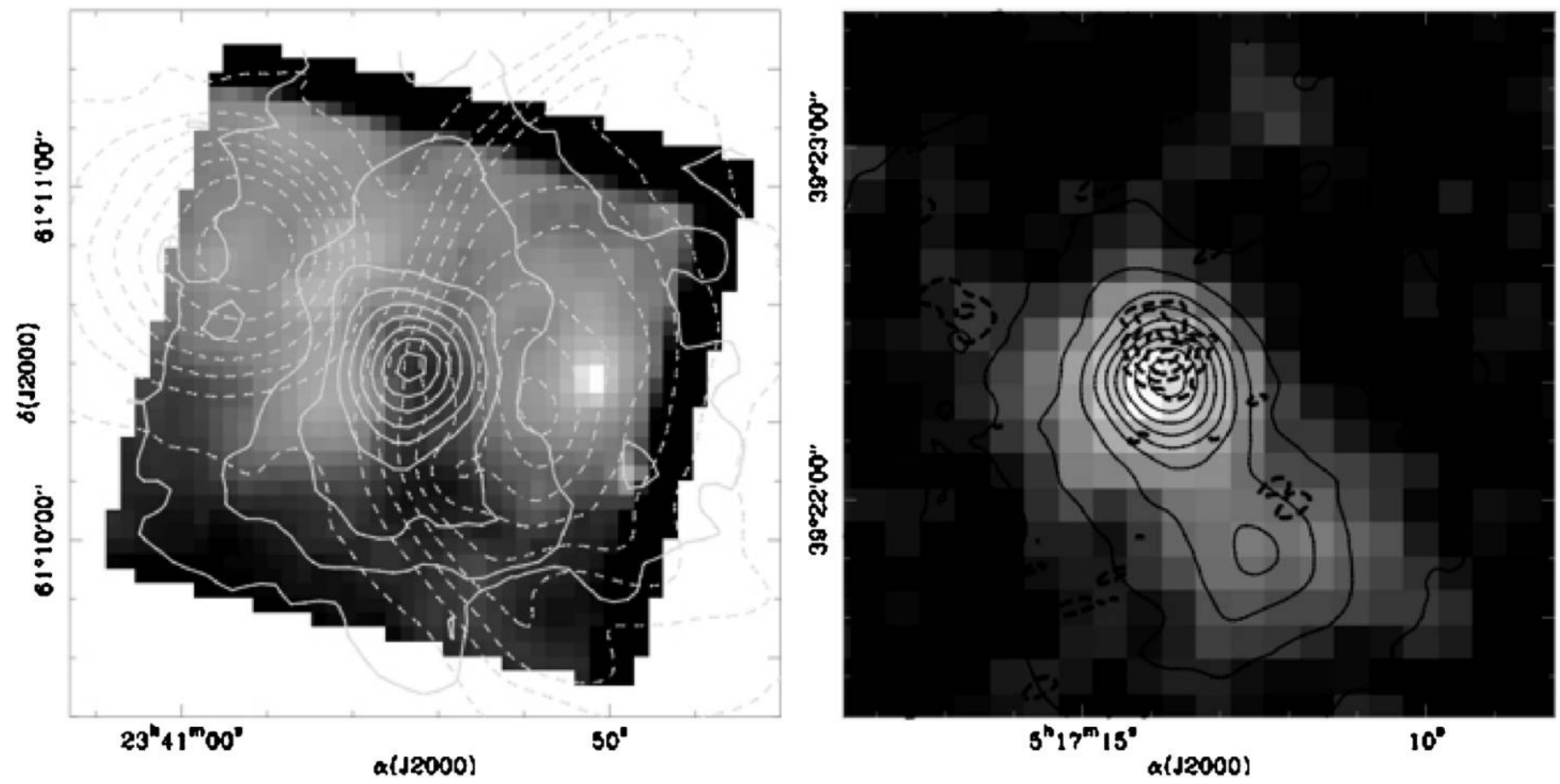

FIG. 7.-Mid-IR $21 \mu \mathrm{m}$ MSX images for a candidate precursor of a Hot Core (IRAS23385 + 6053, left), and a UC H II (IRAS05137 + 3919, right). Full and dashed contours represent the millimeter (cold dust) and radio (jet) continuum emission (from Molinari et al. 2008). Hi-GAL will complement these with similar resolution FIR data. See the electronic edition of the PASP for a color version of this figure.

The abundance of high-mass YSOs per mass bin in the various evolutionary phases will provide an estimate of the duration of each phase. This timeline can be directly compared with the predictions of various models, and together with the YSO mass function, will be used to infer the SFR. As an example, using current estimates for SFR and IMF (McKee \& Williams 1997; Kroupa 2001), and a $10^{5}$ yr period to assemble a massive star (McKee \& Tan 2003), we expect $\geq 1000$ FIR/submillimeter objects with $M>15 M_{\odot}$ in the Galaxy, and only $\sim 180$ objects with $M>50 M_{\odot}$. Recent millimeter surveys in the Cyg X region (Motte et al. 2007) confirm the rarity of such massive precursors and strengthen the need for a systematic unbiased search.

\subsection{Bridging the Gap between Global and Local Star Formation}

Galactic phenomenology currently invokes an indeterminate mixture of spontaneous and triggered star formation. Triggering agents include radiation pressure from OB stars (Sugitani et al. 1989), compression by expanding $\mathrm{H}$ II regions (Elmegreen \& Lada 1977; Deharveng et al. 2005), or fragmentation of supershells by multiple supernovae in OB associations (McCray \& Kafatos 1987). On larger scales we still do not know if spiral density waves actively induce star formation (e.g., Elmegreen 2002) or simply assemble star-forming regions, with local feed- back and triggering becoming more important within the arms (Sleath \& Alexander 1996). The mean SFE of a galaxy can increase (up to 50 times) in starbursts (Sanders et al. 1991) and galaxy mergers due to strong feedback effects, a process observed in miniature in Galactic star-forming regions (e.g., Moore et al. 2007). Whether the IMF depends on local triggering and other environmental factors is unclear.

Hi-GAL will enable quantitative analysis based on basic observables - the luminosity functions of YSOs, the mass function of dense star-forming structures and quiescent clouds. Hi-GAL will provide the essential context of high-mass star formation, as it relates to molecular gas, $\mathrm{H}$ I gas, stars, $\mathrm{H}$ II regions, OB associations, supernova remnants (SNRs), and spiral arms. Theoretical models and numerical simulations will be tested in multiple ways. We will discover whether a local triggering agent is necessary for high-mass star formation or if a spiral arm is sufficient, clarifying the differences between spontaneous and triggered star formation. We will quantify the relationship between the interaction strength (estimated using available data from ancillary surveys) and the resulting increase in SFE above the spontaneous rate. By locally relating the SFR to the properties of the ISM we will probe star-formation thresholds as a function of environment and spatial scale, and possibly unveil the mechanism giving rise to global Schmidt-like scaling laws. We will determine the dominant physical process underlying triggering. 


\subsection{Serendipitous Science}

The 5 band FIR images and source catalogs provided by Hi-GAL will allow research in many fields that can only be partially anticipated. A detailed description of the specific outcomes in all these fields goes beyond the scope of the present paper. These aspects will of course be the object of dedicated publications to be released in due time. In the following we list some of the possible byproducts of Hi-GAL:

1. An input catalog for ALMA: we expect to detect some 200-400 objects per tile, most of which will mark very cold objects to be studied at all possible wavelengths;

2. cComplete characterization of the Galactic foreground in the far-IR and submillimeter, critical for the correct interpretation and modeling of cosmological backgrounds;

3. Dust formation and destruction in SNRs;

4. Debris dust disks around main-sequence stars, with unbiased statistics on frequency and mass as a function of star age;

5. Evolution of dust properties, especially around AGB stars, the factories of cosmic dust;

6. Detection of detached dust shells around first ascent giant stars to investigate missing mass in AGB envelopes;

7. Detection of multiple shells around AGB stars, post-AGB objects, and planetary nebulae, as well as around various classes of interacting binaries;

8. Detection of ejecta shells and swept-up ISM bubbles around massive stars, providing a complete census of WR and LBV stars;
9. Extinction maps to aid in correcting near-IR galactic star counts;

10. Detection of Solar System objects via comparison of cross-linked rasters: in particular the detection of asteroids will be very interesting for studies of the albedo;

11. Nearby low-mass SFRs in the GP: Herschel will detect many nearby star-forming regions and individual YSOs.

\section{HI-GAL AND ITS PLACE IN THE CONTEXT OF THE MULTIWAVELENGTH MILKY WAY}

PACS and SPIRE are unique in tracing the peak of the spectral energy distribution (SED) of cold dust and, hence, temperatures and luminosities of both star-forming complexes and the ISM, at resolutions unmatched by any previous instruments. However, the full potential of the Hi-GAL survey will be realized in the context of the other unbiased Galactic plane surveys which are shaping our understanding of the Galactic ecosystem. A suite of surveys in the mid- and far-infrared continuum, ISOGAL (Omont et al. 2003), MSX (Price et al. 2001), GLIMPSE (Benjamin et al. 2003), MIPSGAL (Carey et al. 2009), and IRAS (Neugebauer et al. 1984), has been and will be complemented by surveys in the submillimeter and millimeter spectral range including the BGPS survey with Bolocam at $1.1 \mathrm{~mm}$ (Rosolowsky et al. 2009), the ATLASGAL survey currently underway with the LaBoCa camera at APEX (Schuller et al. 2009), and the SCUBA2 JPS survey, beginning in 2010 (Fig. 8).

BLAST and AKARI have wavelength coverage and resolution not too different form PACS and SPIRE. However, BLAST

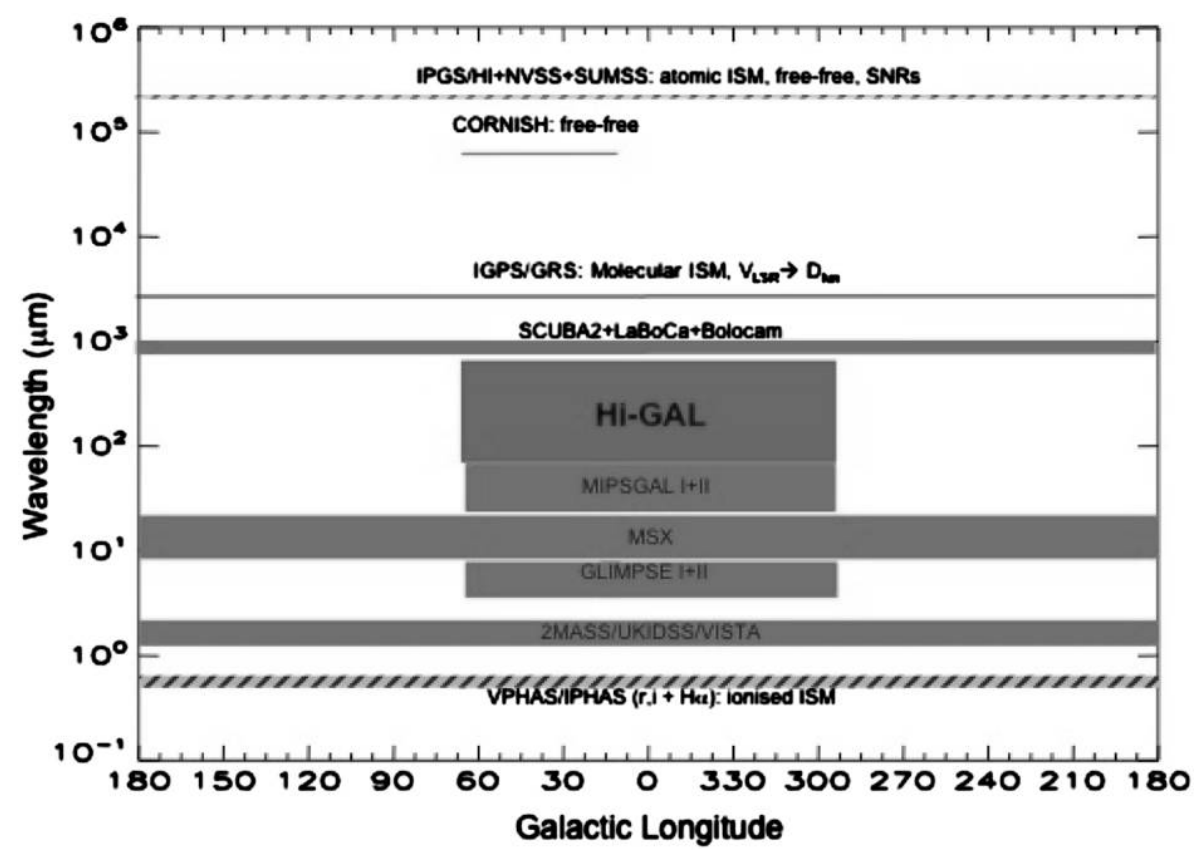

FIG. 8.-Wavelength-longitude coverage plot for photometric and spectroscopic existing or planned GP surveys for the next decade. Hi-GAL fills the critical gap where the ISM dust emission peaks, between MIPSGAL and the SCUBA2/LaBoCa/Bolocam surveys. See the electronic edition of the PASP for a color version of this figure. 
has been used to map limited portions of the GP while results from AKARI photometric imaging of the GP are not yet found in the literature.

Hi-GAL will be the scientific keystone of this suite of surveys, completing the continuous coverage of the dust continuum over 3 orders of magnitude in wavelength at sub-30" resolution, and allowing the measurement of dust temperatures and luminosity over the inner Galactic Plane.

An extensive plan for radio spectroscopic cross-correlation and follow-up of the Hi-GAL survey has been devised. The top priority is for radio spectroscopic observations to obtain distance estimates for detected sources and structures. $\mathrm{CO}$ and ${ }^{13} \mathrm{CO}$ data at subarcminute spatial resolution are available via the IGPS for the first Galactic Quadrant (QI); lower resolution CO data are available from the NANTEN survey (QIV). Should $\mathrm{CO}$ resolution not be sufficient, e.g., in case of very cold cores with $\mathrm{CO}$ depletion, we plan extensive follow-up programs (e.g., $\mathrm{N}_{2} \mathrm{H}^{+}, \mathrm{NH}_{3}$ ) at the Mopra $22 \mathrm{~m}$ antenna (Australia) for QIV, and at the Green Bank, Effelsberg, Medicina, and Onsala telescopes for QI. HI is also available for QI and IV at sufficient resolution from the IGPS to help resolve the distance ambiguity in the inner Galaxy (Busfield et al. 2006). $\mathrm{H} \alpha$ VPHAS + IPHAS surveys will be also used.

In addition to the kinematic distance estimates, we plan to use NANTEN2, Mopra, and APEX for detailed multiline studies of evolution-sensitive chemical tracers $\left(\mathrm{N}_{2} \mathrm{H}^{+}, \mathrm{NH}_{3}, \mathrm{CS}, \mathrm{HCN}\right.$, $\mathrm{CH}_{3} \mathrm{OH}, \mathrm{CH}_{3} \mathrm{CN}$, etc.) toward clouds and objects discovered by Hi-GAL. Hi-GAL catalogs will be the primary source of major future high spatial resolution follow-ups in the submillimeter with ALMA; in the meantime, such programs will be attempted using the SMA interferometer through Legacy class proposals.

\section{DATA PROCESSING AND PRODUCTS}

It is relatively easy to translate our scientific goals into a clear set of requirements on the data processing: we require that the dust continuum emission be detectable, and accurately measurable, at all bands over the broadest range in signal levels (down to the confusion limit) and spatial scales. The observing strategy is carefully designed to that effect, but ensuring that this information is properly extracted from the data stream over a $240 \mathrm{deg}^{2}$ area is a formidable challenge. We will use the Herschel Interactive Processing Environment (HIPE) for all those processing steps dealing with fundamental instrumental calibration and issues, but we anticipate areas where a dedicated set of specialized tools can take advantage of the homogeneous observing strategy and deliver higher quality results compared to the standard pipeline products: pointing, refining, map-making, source extraction, and photometry are examples.

We will make available a set of data products that will include maps and compact source catalogs at the five Hi-GAL wavelengths. These products will be made available after the end of observations (EoO) for the entire survey via incremental releases.

Improved reprocessed maps and source catalogs will be subsequently made public, together with a first release for an extended source catalog. All public deliveries will be accompanied by an Explanatory Supplement.

In addition to this minimum set of products, quite standard for any large-scale survey like Hi-GAL, we plan to make available to the community a set of scientific value-added products which will be created during our scientific analysis, including band-merged catalogs integrated with data from continuum surveys at adjacent wavelength, color maps, and source-subtracted maps. The public access to this final set of products is foreseen for $\mathrm{EoO}+42$ months.

\section{CONCLUSIONS}

Hi-GAL is an Open Time Key Project to be performed with the $3.5 \mathrm{~m}$ orbiting Herschel telescope, to map photometrically the inner Milky Way $\left(|l|<60^{\circ},|b|<1^{\circ}\right)$ in five wavebands between $70 \mu \mathrm{m}$ and $500 \mu \mathrm{m}$ simultaneously, using $\sim 350 \mathrm{hr}$ of observing time. The unique combination of survey speed, high sensitivity, high spatial resolution, and wavelength coverage (right across the peak of the dust emission) make Hi-GAL the first dedicated project to study the early phases of GMCand high-mass star formation in the Galaxy, with a legacy value similar to the IRAS mission some 20 years ago. The outcomes of Hi-GAL will consist of source lists and images to be released in due course after EoO.

We are grateful to all the people who made the building and launch of Herschel such a success. In particular, ESA and the Herschel Project Scientist G. Pilbratt, and the instrument teams of PACS and SPIRE, magnificently led by A. Poglitsch (MPE, Garching) and M. Griffin (University of Cardiff).

\section{REFERENCES}

Abergel, A., Boulanger, F., Mizuno, A., \& Fukui, Y. 1994, ApJ, 423, L59

Andre, P., Ward-Thompson, D., \& Barsony, M. 1993, ApJ, 406, 122 Beltrán, M. T., Brand, J., \& Cesaroni, R., et al. 2006, A\&A, 447, 221 Benjamin, R. A., Churchwell, E., \& Babler, B. L.,et al. 2003, PASP, 115,953

Bernard, J. P., Boulanger, F., \& Puget, J. L. 1993, A\&A, 277, 609

Blitz, L. 1997, in IAU Symp. 170 CO: 25 years of Millimeter Wave Spectroscopy, ed. Latter, W. B. et al., 11
Bot, C., Boulanger, F., Rubio, M., \& Rantakyro, F. 2007, A\&A, 471, 103

Busfield, A. L., Purcell, C. R., \& Hoare, M. G., et al. 2006, MNRAS, 366, 1096

Carey, S. J., Feldman, P. A., \& Redman, R. O., et al. 2000, ApJ, 543, L157

Carey, S. J., Noriega-Crespo, A., \& Mizuno, D. R., et al. 2009, PASP, 121,76

Deharveng, L., Zavagno, A., \& Caplan, J. 2005, A\&A, 433, 565 
Désert, F., Macías-Pérez, J. F., \& Mayet, F., et al. 2008, A\&A, 481, 411

Douglas, K., \& Taylor, A. R. 2007, ApJ, 659, 426

Egan, M. P., Shipman, R. F., \& Price, S. D., et al. 1998, ApJ, 494, L199

Elmegreen, B. G. 2000, ApJ, 530, 277

- 2002, ApJ, 577, 206

Elmegreen, B. G., \& Lada, C. J. 1977, ApJ, 214, 725

Evans, N. J., Shirley, Y. L., Mueller, K. E., \& Knez, C. 2002, in ASP Conf. Ser. 267, Hot Star Workshop III: The Earliest Phases of Massive Star Birth, ed. Crowther, P., 17

Flower, D. R., Pineau Des Forêts, G., \& Walmsley, C. M. 2005, A\&A, 436, 933

Gibson, S. J., Taylor, A. R., Higgs, L. A., Brunt, C. M., \& Dewdney, P. E. 2005, ApJ, 626, 214

Gibson, S. J., Taylor, A. R., \& Higgs, L. A., et al. 2000, ApJ, 540, 851

Glover, S. C. O., \& Mac Low, M. 2007, ApJ, 659, 1317

Goldsmith, P. F., \& Li, D. 2005, ApJ, 622, 938

Griffin, M., Ade, P., \& André, P., et al. 2009, in EAS Publication Series, Pagani, L., \& Gerin, M., 34, 33

Hartmann, L., Ballesteros-Paredes, J., \& Bergin, E. A. 2001, ApJ, 562,852

Hill, T., Burton, M. G., \& Minier, V., et al. 2005, MNRAS, 363, 405

Hoare, M. G., Lumsden, S. L., \& Oudmaijer, R. D., et al. 2004, in ASP Conf. Ser. 317, Milky Way Surveys: The Structure and Evolution of our Galaxy, ed. Clemens, D., Shah, R., \& Brainerd, T. 156

Juvela, M., \& Padoan, P. 2003, A\&A, 397, 201

Kim, K., Lee, J., \& Koo, B. C. 1999, ApJ, 523, 306

Klaassen, P. D., Plume, R., Gibson, S. J., Taylor, A. R., \& Brunt, C. M. 2005, ApJ, 631, 1001

Kroupa, P. 2001, MNRAS, 322, 231

Kurtz, S., Cesaroni, R., Churchwell, E., Hofner, P., \& Walmsley, C. M. 2000, Protostars and Planets IV, 299

Lada, C. J., \& Wilking, B. A. 1984, ApJ, 287, 610

Lagache, G., Abergel, A., Boulanger, F., \& Puget, J. 1998, A\&A, 333,709

Leisawitz, D., Bash, F. N., \& Thaddeus, P. 1989, ApJS, 70, 731

Li, D., \& Goldsmith, P. F. 2003, ApJ, 585, 823

McCray, R., \& Kafatos, M. 1987, ApJ, 317, 190

McKee, C. F., \& Tan, J. C. 2003, ApJ, 585, 850

McKee, C. F., \& Williams, J. P. 1997, ApJ, 476, 144

Molinari, S., Brand, J., Cesaroni, R., \& Palla, F. 1996, A\&A, 308, 573

Molinari, S., Pezzuto, S., \& Cesaroni, R., et al. 2008, A\&A, 481, 345

Molinari, S., Testi, L., Brand, J., Cesaroni, R., \& Palla, F. 1998, ApJ, 505, L39
Moore, T. J. T., Bretherton, D. E., \& Fujiyoshi, T., et al. 2007, MNRAS, 379,663

Motte, F., Bontemps, S., \& Schilke, P., et al. 2007, A\&A, 476, 1243

Murakami, H., Baba, H., \& Barthel, P., et al. 2007, PASJ, 59, 369

Neugebauer, G., \& Soifer, B. T., Beichman, C. A., et al. 1984, Science, 224, 14

Olmi, L., Ade, P., \& Anglés-Alcázar, D., et al. 2009, ApJ, 1836

Omont, A., Gilmore, G. F., \& Alard, C., et al. 2003, A\&A, 403, 975

Padoan, P., \& Nordlund, Å. 1999, ApJ, 526, 279

Palla, F., \& Galli, D. 1997, ApJ, 476, L35

Pascale, E., Ade, P. A. R., \& Bock, J. J., et al. 2008, ApJ, 681, 400

Poglitsch, A., Waelkens, C., \& Bauer, O., et al. 2008, Proc. SPIE, 7010, 701005

Preibisch, T., Ossenkopf, V., Yorke, H., \& Henning, T. 1993, A\&A, 279, 577

Price, S. D., Egan, M. P., Carey, S. J., Mizuno, D. R., \& Kuchar, T. A. 2001, AJ, 121, 2819

Rathborne, J. M., Jackson, J. M., \& Simon, R. 2006, ApJ, 641, 389

Reach, W. T., Dwek, E., \& Fixsen, D. J., et al. 1995, ApJ, 451, 188

Rosolowsky, E., Dunham, M. K., \& Ginsburg, A., et al. 2009, ArXiv e-prints (2009arXiv0909.2871R)

Roy, A., Ade, P., \& Bock, J., et al. 2010, ApJ, in press

Sanders, D. B., Scoville, N. Z., \& Soifer, B. T. 1991, ApJ, 370, 158

Schuller, F., Menten, K. M., \& Contreras, Y., et al. 2009, A\&A, 504,415

Shu, F. H., Adams, F. C., \& Lizano, S. 1987, ARA\&A, 25, 23

Simon, R., Rathborne, J. M., Shah, R. Y., Jackson, J. M., \& Chambers, E. T. 2006, ApJ, 653, 1325

Sleath, J. P., \& Alexander, P. 1996, MNRAS, 283, 358

Sodroski, T. J., Bennett, C., \& Boggess, N., et al. 1994, ApJ, 428, 638

Sridharan, T. K., Beuther, H., Schilke, P., Menten, K. M., \& Wyrowski, F. 2002, ApJ, 566, 931

Stahler, S. W., Palla, F., Ho, P. T. P. 2000, Protostars and Planets IV, 327

Stamatellos, D., Whitworth, A. P., André, P., \& Ward-Thompson, D. 2004, A\&A, 420, 1009

Stepnik, B., Abergel, A., \& Bernard, J., et al. 2003, A\&A, 398, 551

Stone, J. M., Ostriker, E. C., \& Gammie, C. F. 1998, ApJ, 508, L99

Sugitani, K., Fukui, Y., Mizuni, A., \& Ohashi, N. 1989, ApJ, 342, L87

Yorke, H. W., \& Sonnhalter, C. 2002, ApJ, 569, 846

Zinnecker, H., \& Yorke, H. W. 2007, ARA\&A, 45, 481 